\title{
UNA NUEVA COMBINACIÓN EN EL GÉNERO ALLOTOONIA (APOCYNACEAE, APOCYNOIDEAE, ECHITEAE)
}

\author{
J. Francisco Morales \\ Instituto Nacional de Biodiversidad, apdo. 22-3100, Santo Domingo, Heredia, Costa Rica
}

\author{
Justin K. Williams
}

Department of Biological Sciences, Sam Houston State University, Huntsville, TX 77341-2116, U.S.A.

\begin{abstract}
A new combination, Allotoonia woodsoniana (Monac.) J.F. Morales \& J.K. Williams, is published to replace the illegitimate name $A$. parviflora (Sessé \& Moc.) J.F. Morales \& J.K. Williams.
\end{abstract}

Resumen. La nueva combinación Allotoonia woodsoniana (Monac.) J.F. Morales \& J.K. Williams reemplaza al nombre ilegítimo A. parviflora (Sessé \& Moc.) J.F. Morales \& J.K. Williams.

Palabras Clave / Key words: Apocynaceae, Apocynoideae, Echiteae, México, Mesoamerica.

El género Allotoonia (Apocynaceae, Apocynoideae, Echiteae) fue descrito y publicado recientemente (Morales \& Williams 2004) para los miembros de Echites subgénero Pseudechites (Woodson 1936), dado que un análisis cladístico realizado por Williams (2004) demostró que la circunscripción tradicional de Echites es polifilética. Por tanto, una nueva descripción genérica y cinco nuevas combinaciones fueron publicadas por Morales \& Williams (2004). Sin embargo, la combinación Allotoonia parviflora (Sessé \& Moc.) J.F. Morales \& J.K. Williams es superflua, debido a que se basa en un nombre ilegítimo (Echites parviflora Sessé \& Moc.) y en una interpretación errónea del artículo 58 del Código Internacional de Nomenclatura Botánica (Greuter et al. 2000).

El artículo 58 establece que el epíteto de un nombre ilegítimo puede usarse en una combinación diferente (pudiendo conservar incluso el mismo tipo), si no está disponible ningún otro epíteto de un nombre que tenga prioridad a ese rango. En el caso de Echites parviflora Sessé \& Moc. (1888), existe otro nombre disponible publicado posteriormente para el mismo taxon: E. woodsoniana Monac. (1959), el cual tiene prioridad y debe ser usado como basiónimo.

Puesto que la combinación Allotoonia parviflora (Sessé \& Moc.) J.F. Morales \& J.K. Williams es ilegítima, a continuación se publica una nueva combinación para el mismo taxon, basada en el otro nombre disponible.
Allotoonia woodsoniana (Monac.) J.F. Morales \& J.K. Williams, comb. nova

Basiónimo: Echites woodsoniana Monac., Bull. Torrey Bot. Club. 86: 245-247, f.1. 1959. Prestonia woodsoniana (Monac.) A.H. Gentry, Ann. Missouri Bot. Gard. 70: 205. 1983.

Tipo: México; Michoacán, Apatzingán, 13 oct 1939 (fl), Hinton 15325 [Holotipo: NY, isotipos: GH, MO, NY, TEX, US (2 láminas)].

Echites parviflora Sessé \& Moc., Naturaleza(México), ser. 2, 1. app. 28. 1888, nom. illeg., non Roxb. 1832. Allotoonia parviflora (Sessé \& Moc.) J.F. Morales \& J.K. Williams, Sida 21: 148. 2004, nom. illeg.

Tipo: México; Michoacán, Apatzingán, sin fecha (fl), Sessé \& Moçiño 5068 [Lectotipo, designado por Williams 2002: MA, foto F (neg. 41238); isolectotipo: F].

Agradecimientos. Se agradece la colaboración de Michael Grayum (MO), por haber corroborado la correcta aplicación del artículo 58 del Código Internacional de Nomenclatura Botánica.

\section{LiTERATURA CITADA}

Greuter, W., J. McNeill, F.R. Barrie, H.-M. Burdet, V. Demoulin, T.S. Filgueiras, D.H. Nicolson, P.C. Silva, J.E. Skog, P. Trehane, N.J. Turland \& D.L. Hawksworth. 2000. International Code of Botanical Nomenclature (St. Louis Code), Regnum Vegetabile 138. Koeltz Scientific Books, Köni gstein.

Morales, J.F. \& J.K. Williams. 2004. Allotoonia, a new neotropical genus of Apocynaceae based on a 
subgeneric segregate of Echites. Sida 21: 133-158.

Williams, J.K. 2002. Polyphyly of the genus Echites (Apocynaceae: Apocynoideae: Echiteae): evidence based on a morphological cladistic analysis. Sida
21(1): 117-131.

Woodson, R.E. 1936. Studies in the Apocynaceae. IV. The American genera of Echitoideae. Ann. Missouri Bot. Gard. 23: 169-438. 